\title{
Integrating artificial neural network and classical methods for unsupervised classification of optical remote sensing data
}

\author{
Ahmed AK Tahir
}

\begin{abstract}
A novel system named unsupervised multiple classifier system (UMCS) for unsupervised classification of optical remote sensing data is presented. The system is based on integrating two or more individual classifiers. A new dynamic selection-based method is developed for integrating the decisions of the individual classifiers. It is based on competition distance arranged in a table named class-distance map (CDM) associated to each individual classifier. These maps are derived from the class-to-class-distance measures which represent the distances between each class and the remaining classes for each individual classifier. Three individual classifiers are used for the development of the system, K-means and K-medians clustering of the classical approach and Kohonen network of the artificial neural network approach. The system is applied to ETM + images of an area North to Mosul dam in northern part of Iraq. To show the significance of increasing the number of individual classifiers, the application covered three modes, UMCS@, UMCS\#, and UMCS*.In UMCS@, K-means and Kohonen are used as individual classifiers. In UMCS\#, K-medians and Kohonen are used as individual classifiers. In UMCS*, K-means, K-medians and Kohonen are used as individual classifiers. The performance of the system for the three modes is evaluated by comparing the outputs of individual classifiers to the outputs of UMCSs using test data extracted by visual interpretation of color composite images. The evaluation has shown that the performance of the system with all three modes outrages the performance of the individual classifiers. However, the improvement in the class and average accuracy for UMCS* was significant compared to the improvements made by UMCS@, and UMCS\#. For UMCS*, the accuracy of all classes were improved over the accuracy achieved by each of the individual classifiers and the average improvements reached (4.27, 3.70, and 6.41\%) over the average accuracy achieved by K-means, K-medians and Kohonen respectively. These improvements correspond to areas of 3.37, 2.92 and $5.1 \mathrm{~km}^{2}$ respectively. While the average improvements achieved by UMCS@ and UMCS\#, respectively, compared to their individual classifiers were (0.77 and 2.79\%) and (0.829 and 2.92\%) which correspond to (0.61 and $\left.2.2 \mathrm{~km}^{2}\right)$ and $(0.65$ and $2.3 \mathrm{~km}^{2}$ ) respectively.
\end{abstract}

\section{Introduction}

Unsupervised classification of remotely sensed data is a technique of classifying image pixels into classes based on statistics without pre-defined training data. This means that the technique is of potential importance when training data representing the available classes is not available. Unsupervised classification is also important for providing a preliminary overview of image classes and more often it is used in the hybrid approach of image classification $[1,2]$.

Correspondence: ahmdi@uod.ac

Department of Computer Science, Faculty of Science, Duhok University, Duhok, Kurdistan Region, Iraq
Several methods of unsupervised classification using classical or neural network approaches have been developed and used consistently in the field of remote sensing. The most commonly used of the classical approach is K-means clustering algorithm [3] while Kohonen network is the most commonly used one of the artificial neural network approach [4]. So far many research works have conducted to improve the accuracy of the unsupervised classifiers. Examples of these works are the use of Kohonen classifier as a pre-stage to improve the results of clustering algorithms such as agglomerative hierarchical clustering, K-means and threshold-based clustering algorithms [5-7].

\section{实}


In those works one algorithm was used as a pre-stage to improve the classification results of another algorithm. That is, the final decision is made according to only one classifier's decision. Methods involving a simultaneous use of more than one classifier in the so-called multiple classifier system (MCS) which is very common in the approach of supervised classification have not been conducted in the unsupervised classification of optical remote sensing data. See for example [8-10] for some of the MCS schemes form supervised classification of remote sensing data. The idea of MCS is based on performing more than two classifiers and integrating their decisions according to some prior or posterior knowledge concerning the output classes to reach the final decision. Prior knowledge is estimated from training data concerning the output classes while posterior knowledge, in general, represents the outputs of the individual classifiers. The operation of integration is done in one of two strategies, either by combining the outputs of the individual classifiers or by selecting one of the individual classifiers outputs. Many methods of integration have been developed for the implementation of MCS in the supervised approach of classification. Examples of combined-based methods of integration are the majority voting rule, which assigns the label scored by majority of the classifiers to the test sample [9] and Belief function, which is knowledge-based method. It is based on the probability estimation provided by the confusion matrix derived from training data set [11]. Examples of the dynamic classifier selection-based method of integration are classifier rank (CR) approach, which takes the decision of the classifier that correctly classifies most of the training samples neighboring the test sample [12] and the local ranking (LR) which is based on ranking the individual classifiers for each class according to the mapping accuracy (MA) of the classes [8].

In this article, an integrated system of unsupervised classification named unsupervised multiple classifier system (UMCS) is developed using individual classifiers from two different approaches, traditional (classical) and artificial neural network. The system is based on new integration method of the dynamic classifier selectionbased type. This method is based on class-distance maps (CDM) for the individual classifiers as the measure upon which the final decision is selected. The CDM of each individual classifier is generated from the measure of $\mathrm{Eu}$ clidean distances between each class and the remaining classes of that individual classifier, named here as the class-to-class distance measurement (CCDM).

The remaining parts of the article are organized as follows: In the following section, the proposed system is described and detailed explanations of its major modules are given. In section "Results", the results of applying the system to ETM + images are shown and discussed. In section "Posterior interpretation of output classes", posterior interpretation of the classification outputs is done. In section "Individuals and multiple classifiers comparison", comparisons between the output results are made. In section "Evaluation of system performance", the performance of the system is evaluated and finally some concluding remarks are given in the last section.

\section{(UMCS); the proposed system}

In this article, the proposed system of classification is called UMCS to be differentiated from the multiple classifier system (MCS) which is common in supervised classification. It is designed to host three individual unsupervised classifiers and can be adapted to any number on individual classifiers. The scheme of the system for three individual classifiers is shown in Figure 1. Each of the three classifiers, K-means, K-medians and Kohonen is implemented using multi-spectral images yielding three output images. These three output images are then entered to a color unification algorithm (CUA) in order to achieve class-to-class correspondence in the three output images. Finally, the three output images of the (CUA) are integrated using CDM generated from the Euclidean distance measurements between each class and the remaining classes within the classifier, named as (CCDM). The algorithms of color unification and classifier integration method are given in the following sections.

\section{CUA}

In most cases the order of classes resulting from different approaches of unsupervised classification are affected by the way of performing the operation of clustering and the order of data presented to the process of clustering. For instance, in the Kohonen network, the training phase usually starts by giving the initial weights

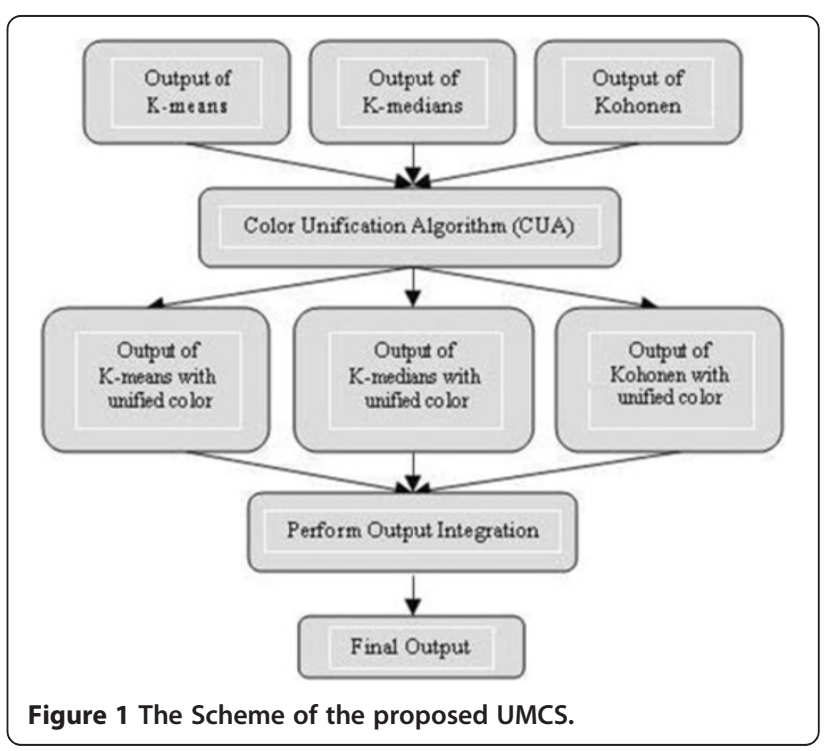


which control the order of the outcome classes. Therefore in order to implement the proposed system, the corresponding classes in the individual classifiers must have the same order. To achieve this step, an algorithm named CUA is developed. The aim of this algorithm is to reorder the classes an all classifiers in order to assign same color to the three nearest classes of the three classifiers. This is done by fixing the order of classes in one classifier as a reference and reordering the classes of the other two classifiers. This algorithm requires the determination of the Euclidean distance between the center of each class in the referenced classifier and the centers of all classes in each of the other two classifiers. The nearest two classes each from one classifier are given the same order (color) of the current class in the reference classifier. Then the operation is repeated until the ordering of all classes in the three classifiers is reached. The algorithm does not require re-calculation of the class centers since these centers are calculated during the implementation of the classifiers. In K-means and K-medians classifiers, the last mean vectors and median vectors upon which the classifier have reached the convergence state represent the centers of the classes. In Kohonen classifier, the weight vectors to the output neurons are taken to be the centers of the classes. The procedures of the algorithm are:

1- Read the centers of the classes for the three classifiers and set the class number $\mathrm{i}=0$.

2- Increase class number $\mathrm{i}=\mathrm{i}+1$.

3- Calculate the Euclidean distance between the mean vector of $C_{i}$ from the reference classifier and the mean vectors of all output classes in the other two classifiers.

$$
\begin{aligned}
& D_{\text {im }}=\left\|C_{i}-P_{m}\right\| \text { for all } m=i,,, k \\
& D_{\text {in }}=\left\|C_{i}-Q_{n}\right\| \text { for all } n=i,,, k
\end{aligned}
$$

Where;

$D_{i m}$ is the Euclidean distance between class $C_{i}$ from the reference classifier and class $P_{m}$ from the second classifier.

$D_{\text {in }}$ is the Euclidean distance between class $C_{i}$ from the reference classifier and class $Q_{n}$ from the third classifier.

|I.|| represents the norm operator.

4- Exchange class order.

Exchange the class order of the second classifier:

if $\left(D_{i j}<D_{i m}\right)$ for all $m=i,, k$ and $j \neq m$

Temp $=P_{j}$

$P_{j}=P_{i}$

$P_{i}=$ Temp

Exchange the class order of the third classifier:

if $\left(D_{i l}<D_{\text {in }}\right)$ for all $n=i,, k$ and $l \neq n$

$$
\begin{aligned}
& \text { Temp }=Q_{l} \\
& Q_{l}=Q_{i} \\
& Q_{i}=\text { Temp }
\end{aligned}
$$

5- Check the convergence of the algorithm.

if $(i<k)$

Go to step 2

else

Go to step 6

6- Stop.

\section{Integration method by CCDM}

As it was mentioned in the introduction, several methods of integrating the outputs of different classifiers are available. These methods were designed for MCS of the supervised type and they require a priori knowledge which most often can be estimated from the training data. For UMCS, the training data are not available and therefore this a priori knowledge cannot be obtained. The method of majority voting may be the only one which can be used to integrate the outputs of unsupervised classifiers since it only requires the final decisions of the three classifiers. However, this rule is influenced by the degree of correlation among the errors made by individual classifiers. When these errors are correlated (all classifiers produce incorrect but similar outputs) it leads to incorrect decision and when these errors are uncorrelated (each classifier produces a unique output) it leads to failure, [9].

In this article, a new method of integration is introduced. It is categorized as a selection-based approach and does not need prior knowledge. It requires a posterior knowledge which can be obtained from the outputs of the three classifiers. This posterior knowledge is the within classifier CCDM which is the measure of Euclidean distance between each class and all of the remaining classes within each individual classifier. This CCDM is then used to generate a table having $\mathrm{N}$ columns and $\mathrm{N}-1$ rows, where $\mathrm{N}$ is the number of classes. The elements under each column represent the distances, stored in ascending way, from the class of that column to all of the remaining classes. For each individual classifier one CDM is generated.

The procedures of implementing the algorithm are given below for UMCS made from three classifiers. It consists of two parts. In the first part, the CDM is generated. In the second part, the process of selecting the final decision is performed. The algorithm can easily be adapted to any number of classifiers. The flowchart of the algorithm is given in Figure 2.

\section{Generation of CDM}

1- Calculate CCDM from all the classes in each classifier using the following equation: 


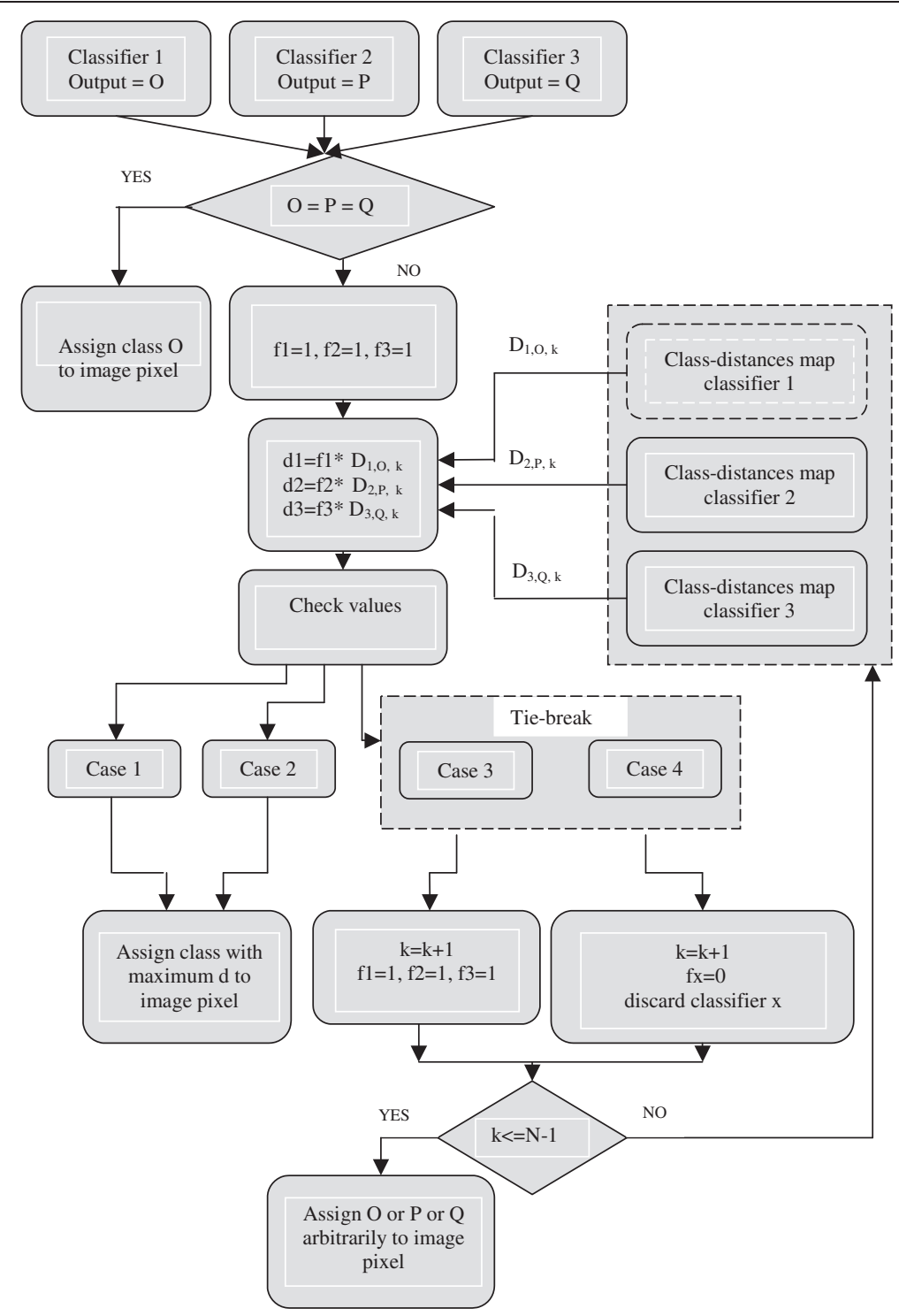

Figure 2 Flowchart of integration method (CCDM).

$$
C C D M_{i j}=\sum\left\|C_{i}-C_{j}\right\|
$$

Where;

$i=1,{ }_{,}, N, j=1,,, N, i \neq j$ and $N$ is the number of classes in each classifier.

|I.|| represents the norm operator.

$\mathrm{C}_{\mathrm{i}}$ is the mean vector of $\mathrm{i}^{\text {th }}$ class and $\mathrm{C}_{\mathrm{j}}$ is the mean vector of $\mathrm{j}^{\text {th }}$ class, both from the same classifier.

For $\mathrm{N}$ classes there will be $\mathrm{N}-1$ distances associated with each class.

2- Generate CDM by sorting the values of CCDM in an ascending way to be used for competition between the individual classifiers. Let these competition distances be represented as $D_{i, j, k}$, where; $\mathrm{i}$ is a subscript refers to the individual classifier, $\mathrm{i}=1,, \mathrm{M}$ and $\mathrm{M}$ is the total number of individual classifiers involved in the UMCS. j refers to the current class, $\mathrm{j}=1, \ldots \mathrm{N}$ and $\mathrm{N}$ is the number of classes which is the same for all individual classifiers.

$\mathrm{k}$ refers to the position of the distances after being sorted in an ascending way. That is, the minimum of all will be at the top of column with position $\mathrm{k}=1$ and the maximum of all will be at the bottom of column with position $\mathrm{k}=\mathrm{N}-1$. During the process of decision selection, these distances at position $\mathrm{k}=1$ will be compared and at tie cases the distances at the next position $\mathrm{k}=2$ will be compared and so on until 
tie break is achieved. If the tie case continued to appear until the last position, which is a rarely occurred case, then it is broken by assigning the class produced by one of the individual classifier arbitrarily. This way of comparison makes the algorithm effective for tie cases as there will almost a zero chance for the occurrence of tie while comparing all the competitive distances. Table 1 shows a model of CDM for classifier i. In this table, each column holds the competition distances associated to each class, starting from class 1 to class $\mathrm{N}$. UMCS of three individual classifiers requires three of these CDM and during the operation of decision selection there will be a competition between these distances in three column each of one CDM.

3- Read the three output images produced by the three individual classifiers pixel by pixel. The values of these pixels represent the class numbers produced by the three classifiers. Let these class numbers are: $\mathrm{O}, \mathrm{P}$ and $\mathrm{Q}$. Then perform the following statements: if $(O=P$ and $O=Q)$ then Assign class $O$ to the pixel of the final output image Else Perform the operation of integrating the decisions made by the three classifiers.

\section{Performing the process of final decision selection}

1- Set record number $k$ to a value of one (the position of the first distance in each of the columns under the output classes) and set three flags (f1, f2, f3) each to a value of one.

2- Read the distances associated to these classes at position $\mathrm{k}\left(\mathrm{D}_{1, \mathrm{O}, \mathrm{k}}, \mathrm{D}_{2, \mathrm{P}, \mathrm{k}}, \mathrm{D}_{3, \mathrm{Q}, \mathrm{k}}\right)$.

3- Compute competitive distances $(\mathrm{d} 1, \mathrm{~d} 2, \mathrm{~d} 3)$ as follows:

$$
\begin{aligned}
& \mathrm{d} 1=\mathrm{f} 1^{*} \mathrm{D}_{1, \mathrm{O}, \mathrm{k}} \\
& \mathrm{d} 2=\mathrm{f}^{*} \mathrm{D}_{2, \mathrm{P}, \mathrm{k}} \\
& \mathrm{d} 3=\mathrm{f}^{*} \mathrm{D}_{3, \mathrm{Q}, \mathrm{k}}
\end{aligned}
$$

\begin{tabular}{|c|c|c|c|c|}
\hline & Class it & Classi2 & $\ldots \ldots$ & Class iN \\
\hline \multirow{6}{*}{$\operatorname{Min}(k=1)$} & $D_{i, 1,1}$ & $D_{i, 2,1}$ & ..... & $D_{i, N, 1}$ \\
\hline & $D_{i, 1,2}$ & $D_{i, 2,2}$ & $\ldots \ldots$ & $D_{i, N, 2}$ \\
\hline & . & . & $\ldots \ldots$ & \\
\hline & . & . & $\ldots \ldots$ & \\
\hline & & . & $\ldots \ldots$ & \\
\hline & & . & $\ldots \ldots$ & \\
\hline $\operatorname{Max}(k=N-1)$ & $D_{i, 1, N-1}$ & $D_{i, 2, N-1}$ & $\ldots \ldots$ & $D_{i, N, N-1}$ \\
\hline
\end{tabular}

Table 1 A model of CDM for classifier i
4- Check the values of these competitive distances to perform one of the following cases for final decision:

Case 1: If these competitive distances are alternatively different, then assign the class with maximum competitive distance to the pixel of the final output image.

Case 2: $\quad$ If any two of these competitive distances have the same value and this value is lower than the competitive distance of the other class, then assign the other class to the pixel of the final output image.

Case 3 (Tie-Break): If the competitive distances for the three classes are all the same, then increase $\mathrm{k}$ by one and go to step 2 to read the next associated distances. If the tie case remained unbroken, then assign one of the classes arbitrarily to the pixel of the final output image.

Case 4 (Tie-Break): If the competitive distances of any two classes have same value and this value is greater than the competitive distance of the other class, then discard the other class from competition by resetting its flag (f) to zero and increase $k$ by one then go to step 2 . Here, the competition will remain between two output classes as far as the tie is not broken and if $\mathrm{k}$ value reached the last record at a position number equals $(\mathrm{m}-1)$ where $m$ is the total number of classes without achieving tiebreak, then assign one of the classes arbitrarily to the pixel of the final output image, otherwise assign the class with maximum competitive distance to the pixel of the final output image.

\section{Results}

The system is applied to ETM + image of an area north to Mosul dam in the northern part of Iraq. The image size is $296 \times 296$ square pixels which is equivalent to an area of $78.85 \mathrm{~km}^{2}$. Standard Kohonen network with $\mathrm{R}=0$ was used (the weights of only the winner neuron are updated). The number of neurons in the input layer was chosen to be 6 which is the number of the available bands 
of ETM + (band1, band2, band3, band4, band5 and band7). The number of neurons in the output layer was chosen to be 8 which are the same as the output classes in K-means and K-medians. However in practice, training Kohonen network usually needs wise determination of the learning rate and the number of cycles. In this article, different values of learning rate and cycle numbers were tried. Consistent results were reached by using initial learning rate of 0.7 with a decrement of $(0.7 / 500)$ at each next cycle where the number of cycles is taken to be 500. Kohonen neural network with this structure is supposed to be closest to K-means than any of the other structures of Kohonen neural network. $\mathrm{K}$-medians clustering is a variation of K-means, however mathematically medians are calculated instead of means, [13].

The selection of standard Kohonen neural network and the K-medians as being closely related to K-means clustering was done in order to show that, to what extend these classifiers can produce different results and to what extend the application of UMCS can be appreciable when individual classifiers of divers differences are chosen.

The system is applied in three modes using different number and combinations of individual classifiers in order to show the influence of increasing the number of individual classifiers on the system accuracy. In the first mode (UMCS@), K-means and Kohonen were used as two individual classifiers. In the second mode (UMCS\#), K-medians and Kohonen were used as two individual classifiers. In the third mode (UMCS*), $\mathrm{K}$-means, K-means and Kohonen were used as three individual classifiers. Figure 3, shows the classification results of K-means, K-means, Kohonen and the three multiple classifiers UMCS@, UMCS\#, and UMCS*. In unsupervised classification usually the number of classes is chosen either arbitrarily or according to the available knowledge of the study area. Here, this number was chosen to be 8 after visual inspection of the color composite images made from different combinations of the available bands.

To show as to what extend the individual classifiers in each MCS agreed or disagreed in their decisions are given in Table 2 . In this table, the percentages of pixels and their equivalent areas for which all the individual classifiers produced the same and different decisions for the three MCS (UMCS@, UMCS\#, and UMCS*) are shown. According to this table the number of pixels for which the individual classifiers have given different results in the case of $\mathrm{UMCS}^{*}$ is greater than those in UMCS@ and UMCS\#. This is an expected result given the fact that increasing the number of individual classifiers will makes more chances of these classifiers first to give different results and second to produce uncorrelated errors, [14].

\section{Posterior interpretation of output classes}

In unsupervised classification the cover types that represent the output classes must be identified after the

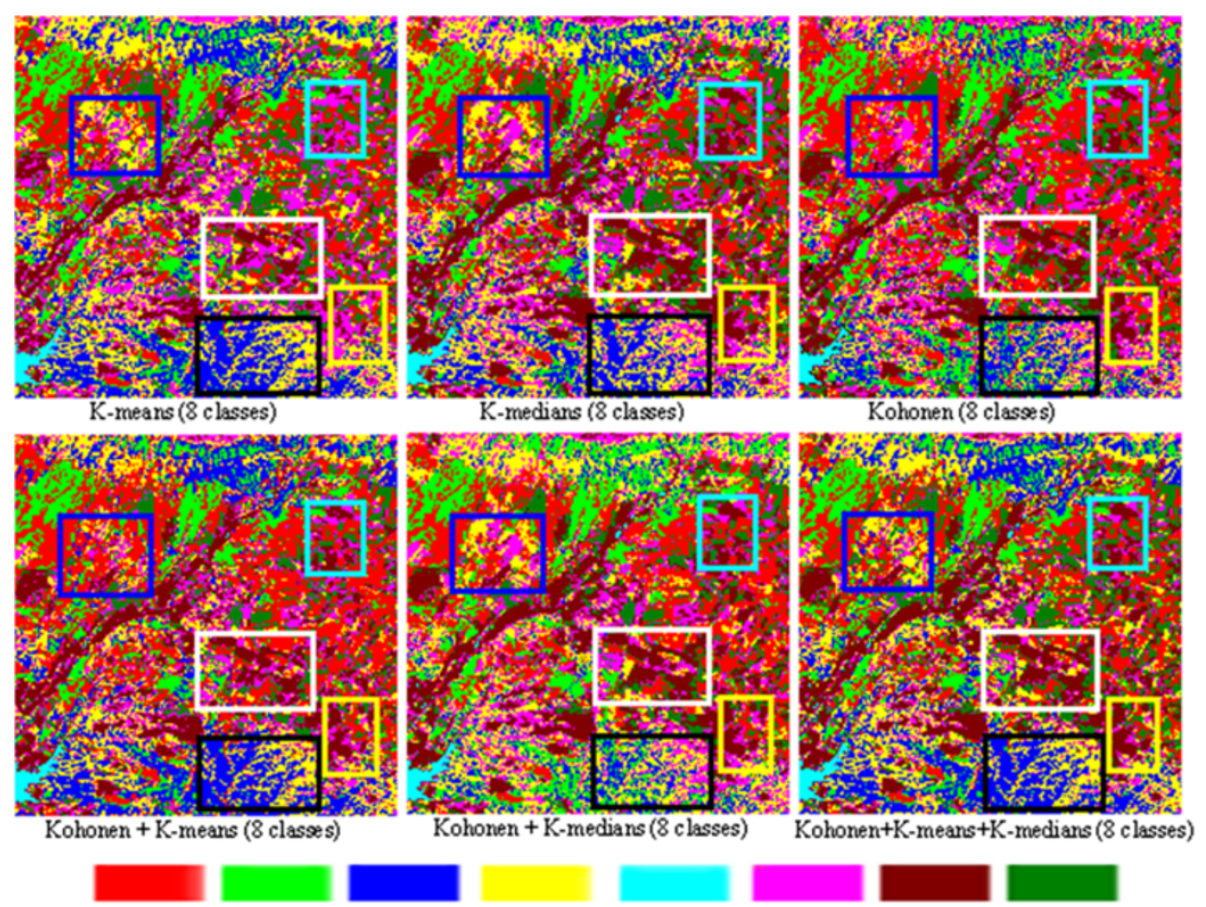

Figure 3 Outputs of six classifiers (K-Means, K-medians, Kohonen, UMCS@, UMCS\# and UMCD*). 
Table 2 Image size percentages and their equivalent areas for which the individual classifier in each of UMCSs produce the same and different decisions

\begin{tabular}{llcc}
\hline & UMCS & Same decision & Different decision \\
\hline UMCS@ & percentage & $69.73 \%$ & $30.27 \%$ \\
& Equivalent area & $54.98 \mathrm{~km}^{2}$ & $23.87 \mathrm{~km}^{2}$ \\
\multirow{2}{*}{ UMCS\# } & percentage & $71.60 \%$ & $28.40 \%$ \\
& Equivalent area & $56.46 \mathrm{~km}^{2}$ & $22.39 \mathrm{~km}^{2}$ \\
\multirow{2}{*}{ UMCS* $^{*}$} & percentage & $60.29 \%$ & $39.71 \%$ \\
& Equivalent area & $47.54 \mathrm{~km}^{2}$ & $31.31 \mathrm{~km}^{2}$ \\
\hline
\end{tabular}

classification. Here, this interpretation was done by comparing the results of the individual classifiers and the multiple classifiers visually to the color composite images of the available bands. Two color composite images were generated using the combinations (band4, band3, band2 as RGB) and (band7, band4, band1 as RGB), Figure 4. First, the interpretation of these color composite images was implemented by comparing the colors in these two color composite images to the spectral properties of the cover types. This is one of the most commonly used methods for remote sensing data interpretation, [4]. Table 3 shows the identities of the output classes after interpretation.

\section{Individuals and multiple classifiers comparison}

To visualize the differences between the outputs of the six classifiers, five areas were localized in rectangles of different colors. These differences can be illustrated for the area in black rectangle. Figure 5 is the zoomed image of the black rectangles for the six classifiers. In the product of $\mathrm{K}$-means the area of this rectangle is dominated by blue and yellow colors, which correspond to (Dry
Table 3 Identities of output classes

\begin{tabular}{lcc}
\hline Class No. & Color & Class type \\
\hline 1 & Red & Less Wet Red Soil \\
2 & Green & Dry Red Soil \\
3 & Blue & Dry Gray Soil \\
4 & Yellow & Wet Red Soil \\
5 & Cyan & Water (Dam) \\
6 & Magenta & Sparse Veg. \\
7 & Dark Red & Dense Veg. \\
8 & Dark Green & Less Dense Veg. \\
\hline
\end{tabular}

Gray Soil) and (Wet Red Soil) cover types respectively. However, the area of blue color within this rectangle for $\mathrm{K}$-means and $\mathrm{K}$-medians are almost the same. In the product of Kohonen, two more colors appeared in this rectangle, the green and some patches of red colors which correspond to (Dry Red Soil) and (Less Wet Red Soil). These variations in the colors within this rectangle indicate that the three individual classifiers can produce different results for the same area. Looking at this rectangle in the UMCS products shows that these colors have been distributed differently for the three UMCS products. For instance in UMCS@ and UMCS* products, the colors and their distributions are almost the same as in K-means. This indicates that the competition between the blue and yellow colors of K-means product on one side and the green, magenta and red colors of the K-medians and Kohonen products on the other side was in favor of K-means classifier. This can be checked by looking at the CDM of the three individual classifiers, Table 4. This table shows that the competitive distance of blue in K-means is higher than the competitive

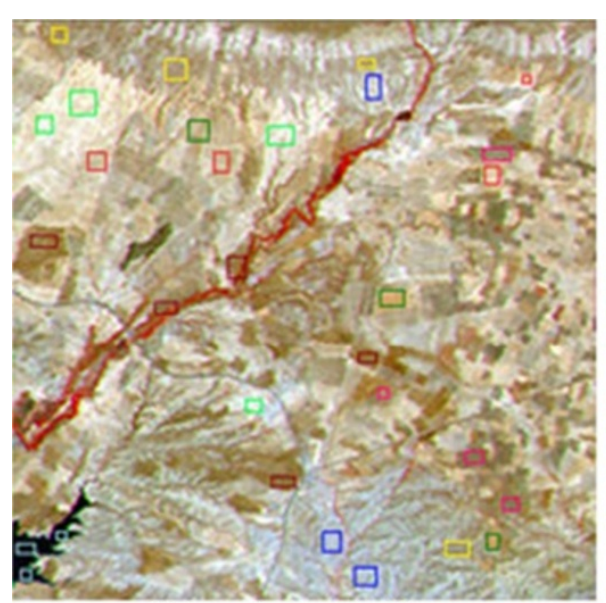

False color composite bands 4,3,2 as RGB

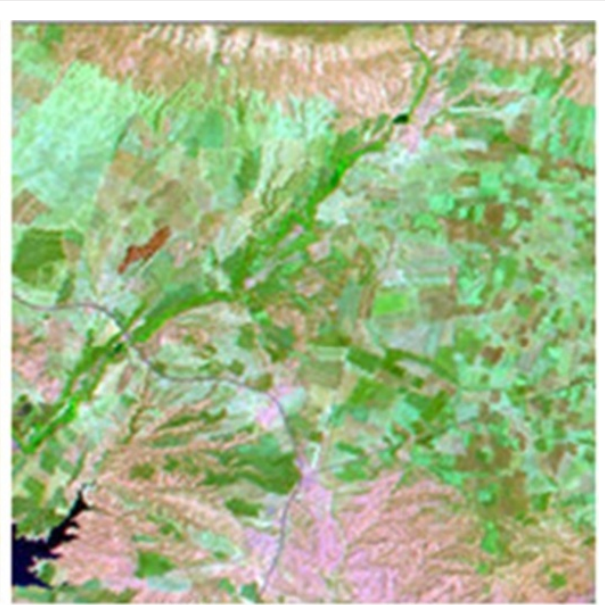

False color composite bands ?,4,1 as RGB

Figure 4 Two color composite images band4, band3, and band2 as RGB and band7, band4, and band1as RGB. 


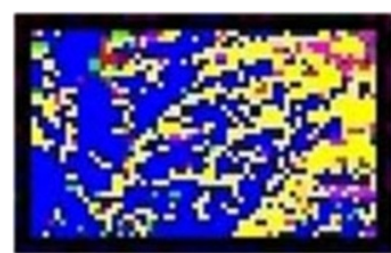

$\mathrm{K}$-means

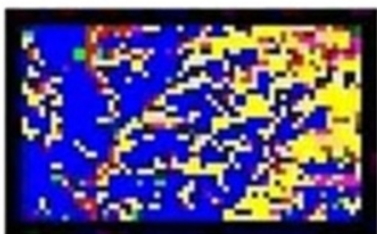

UMCS@

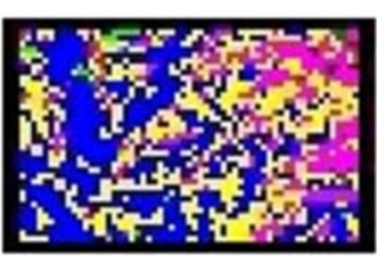

$\mathrm{K}$-medians

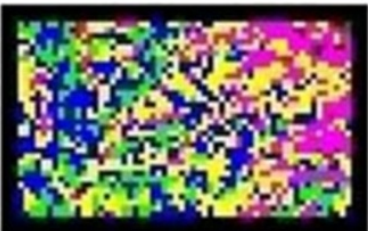

UMCS\#

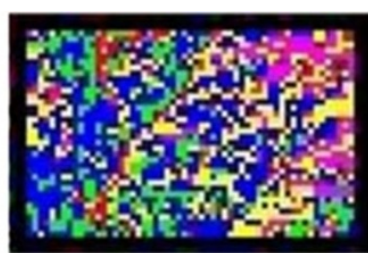

Kohonen

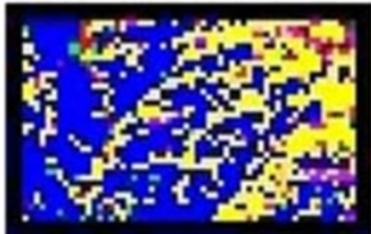

UMCS *

Figure 5 Zoomed details within black rectangles for the individual and multiple classifiers.

distance of green color in Kohonen, therefore blue color will be the winner and will appear in the output of the MCS. On the other hand, the competition distance of yellow colors of the K-means map is greater than the competitive distance of magenta and red colors in the maps of K-medians and Kohonen therefore, in the

Table 4 CDM

\begin{tabular}{|c|c|c|c|c|c|c|c|}
\hline Class 1 & Class 2 & Class 3 & Class 4 & Class 5 & Class 6 & Class 7 & Class 8 \\
\hline Red & Green & Blue & Yellow & Cyan & Magenta & Dark Red & Dark Green \\
\hline \multicolumn{8}{|c|}{ K-means } \\
\hline 25.48 & 28.25 & 26.54 & 24.50 & 116.35 & 25.88 & 27.61 & 24.50 \\
\hline 26.54 & 28.46 & 27.47 & 26.72 & 139.47 & 27.61 & 48.42 & 25.48 \\
\hline 26.72 & 48.62 & 28.25 & 27.47 & 161.32 & 31.23 & 58.26 & 25.88 \\
\hline 28.46 & 53.91 & 44.47 & 31.23 & 168.29 & 48.34 & 73.33 & 44.47 \\
\hline 48.34 & 75.65 & 58.25 & 48.62 & 185.55 & 58.25 & 85.45 & 48.42 \\
\hline 73.33 & 101.42 & 85.45 & 58.26 & 194.07 & 75.65 & 101.42 & 53.91 \\
\hline 185.55 & 212.72 & 194.07 & 168.29 & 212.72 & 139.48 & 116.35 & 161.31 \\
\hline \multicolumn{8}{|c|}{ K-medians } \\
\hline 23.74 & 26.98 & 24.15 & 23.74 & 117.96 & 22.12 & 32.23 & 22.12 \\
\hline 26.52 & 27.63 & 26.98 & 24.15 & 146.05 & 25.98 & 39.51 & 26.52 \\
\hline 27.63 & 41.43 & 29.65 & 25.98 & 155.17 & 32.23 & 57.48 & 29.03 \\
\hline 29.65 & 54.10 & 49.08 & 29.03 & 171.08 & 39.57 & 65.33 & 39.51 \\
\hline 39.57 & 64.36 & 49.48 & 41.43 & 180.65 & 49.48 & 81.16 & 49.08 \\
\hline 65.33 & 92.48 & 81.16 & 57.48 & 193.37 & 64.36 & 92.48 & 54.10 \\
\hline 180.64 & 207.22 & 193.37 & 171.08 & 207.22 & 146.05 & 117.96 & 155.17 \\
\hline \multicolumn{8}{|c|}{ Kohonen } \\
\hline 24.20 & 26.07 & 21.46 & 21.46 & 126.57 & 21.30 & 33.09 & 21.30 \\
\hline 25.78 & 30.90 & 25.78 & 21.91 & 155.90 & 21.91 & 33.54 & 32.20 \\
\hline 30.90 & 43.67 & 26.07 & 24.20 & 156.71 & 33.09 & 54.82 & 33.54 \\
\hline 32.90 & 61.92 & 42.78 & 35.13 & 175.53 & 34.29 & 63.20 & 35.13 \\
\hline 34.29 & 63.37 & 51.94 & 43.67 & 186.84 & 42.78 & 75.76 & 51.94 \\
\hline 63.20 & 93.15 & 75.76 & 54.82 & 195.88 & 61.92 & 93.15 & 63.37 \\
\hline 186.84 & 215.45 & 195.88 & 175.53 & 215.45 & 155.90 & 126.57 & 156.71 \\
\hline
\end{tabular}

A: Class-distance map for K- means. B: Class-distance map for K-medians. C: Class-distance map for Kohonen. 
Table 5 Confusion matrices of the individual and multiple classifiers

\begin{tabular}{|c|c|c|c|c|c|c|c|c|}
\hline \multirow[t]{2}{*}{ Actual classes } & \multicolumn{8}{|c|}{ Produced classes } \\
\hline & Red & Green & Blue & Yellow & Cyan & Magenta & Dark Red & Dark Green \\
\hline \multicolumn{9}{|l|}{ K-means } \\
\hline Red & 290 & 10 & 0 & 7 & 0 & 6 & 5 & 2 \\
\hline Green & 12 & 367 & 12 & 3 & 0 & 2 & 2 & 2 \\
\hline Blue & 0 & 7 & 358 & 13 & 1 & 6 & 14 & 1 \\
\hline Yellow & 8 & 5 & 15 & 355 & 0 & 9 & 7 & 1 \\
\hline Cyan & 0 & 0 & 1 & 1 & 197 & 1 & 0 & 0 \\
\hline Magenta & 10 & 2 & 12 & 5 & 1 & 189 & 1 & 0 \\
\hline Dark Red & 3 & 2 & 10 & 8 & 1 & 11 & 363 & 22 \\
\hline Dark Green & 2 & 0 & 2 & 3 & 0 & 14 & 10 & 229 \\
\hline
\end{tabular}

K-medians

Red

Green

Blue

Yellow

Cyan

Magenta

Dark Red

Dark Green

Kohonen

Red

Green

Blue

Yellow

Cyan

Magenta

Dark Red

Dark Green

UMCS@ (K-means + Kohonen)

Red

Green

Blue

Yellow

Cyan

Magenta

Dark Red

Dark Green

UMCS\# (K-medians + Kohonen)

Red

Green

Blue

Yellow

Cyan

Magenta

$287 \quad 10$

$\begin{array}{ll}0 & 6 \\ 16 & 5 \\ 338 & 18 \\ 13 & 353 \\ 1 & 1 \\ 12 & 6 \\ 1 & 8 \\ 2 & 1\end{array}$

0
0
1
1
196
1
1
0

$\begin{array}{lll}4 & 8 & 5 \\ 2 & 2 & 1 \\ 10 & 13 & 5 \\ 11 & 6 & 4 \\ 1 & 1 & 0 \\ 186 & 6 & 0 \\ 1 & 382 & 19 \\ 1 & 15 & 236\end{array}$


Table 5 Confusion matrices of the individual and multiple classifiers (Continued)

\begin{tabular}{|c|c|c|c|c|c|c|c|c|}
\hline Dark Red & 5 & 3 & 1 & 4 & 1 & 2 & 391 & 13 \\
\hline Dark Green & 6 & 2 & 1 & 1 & 0 & 3 & 11 & 236 \\
\hline \multicolumn{9}{|c|}{ UMCS* (K-means + K-medians + Kohonen) } \\
\hline Red & 296 & 8 & 0 & 2 & 0 & 2 & 5 & 7 \\
\hline Green & 7 & 375 & 10 & 3 & 0 & 2 & 1 & 2 \\
\hline Blue & 0 & 9 & 366 & 12 & 0 & 5 & 6 & 2 \\
\hline Yellow & 7 & 3 & 12 & 363 & 0 & 7 & 5 & 3 \\
\hline Cyan & 0 & 0 & 0 & 0 & 200 & 0 & 0 & 0 \\
\hline Magenta & 6 & 3 & 11 & 5 & 0 & 193 & 1 & 1 \\
\hline Dark Red & 4 & 2 & 2 & 8 & 0 & 5 & 390 & 9 \\
\hline Dark Green & 7 & 4 & 2 & 2 & 0 & 4 & 8 & 233 \\
\hline
\end{tabular}

A: Confusion matrix of K-means. B: Confusion matrix of K-medians. C: Confusion matrix of Kohonen. D: Confusion matrix of UMCS@. E: confusion matrix of UMCS\#. F: Confusion matrix of UMCS*.

output of MCS UMCS* yellow color will be the winner. The same rule can be applied to areas within the other rectangles with the aid of CDM of the individual classifiers.

\section{Evaluation of system performance}

The performance of the system was evaluated by selecting test data from the two color composite images representing the eight classes. The locations of these test data samples were shown as rectangles in the color composite of (Band4, band3, band2 as RGB) of Figure 4. For each class the rectangle is shown in the same color of that class. The numbers of the selected pixels for the classes 1 to 8 respectively were 320,400, 400, 400, 200, 220,420 and 260). This data is then entered to each of the individual classifier (K-means, K-medians and Kohonen) as well as to each of the multiple classifiers (UMCS@, UMCS\# and $\mathrm{UMCS}^{*}$ ).

The MA was measured since this measurement takes into account the pixels that are falsely classified. The confusion matrices of the six classifiers are given in Table 5. In this table, the diagonal elements represent the number of pixels that are correctly classified $\left(P_{\text {corr }}\right)$, the off-diagonal elements in the row of the class represent the number of pixels that are incorrectly classified to other classes, known as omission error $\left(P_{o m}\right)$ and the off-diagonal elements in the column of the class represent pixels that are falsely classified to the current class, known as commission error $\left(P_{\text {com }}\right)$. The MA of the eight classes for each classifier is calculated using the following equation:

$$
M A=\frac{P_{\text {corr }}}{P_{\text {corr }}+P_{\text {om }}+P_{\text {com }}}
$$

Table 6 shows these mapping accuracies for the six classifiers. It can be seen that the MA of all classes are improved by UMCS*, while the MA for some classes were improved and for others were decreased by UMCS@ and UMCS\# classifiers. Table 7 shows the

Table 6 The accuracy mapping of the individual and multiple classifiers

\begin{tabular}{|c|c|c|c|c|c|c|}
\hline Class number & K-means (\%) & K-medians (\%) & Kohonen (\%) & UMCS@ (\%) & UMCS\# (\%) & UMCS* (\%) \\
\hline 1 & 81.69 & 82.00 & 78.14 & 80.67 & 82.15 & 84.33 \\
\hline 2 & 86.15 & 85.02 & 79.09 & 85.68 & 82.32 & 87.41 \\
\hline 3 & 79.20 & 75.95 & 73.69 & 81.59 & 75.51 & 83.75 \\
\hline 4 & 80.68 & 79.32 & 73.56 & 77.60 & 80.00 & 84.02 \\
\hline 5 & 97.04 & 96.07 & 97.53 & 98.01 & 98.01 & 100.0 \\
\hline 6 & 70.26 & 74.40 & 71.00 & 71.59 & 74.90 & 78.77 \\
\hline 7 & 79.08 & 81.10 & 84.54 & 85.65 & 85.93 & 87.44 \\
\hline 8 & 79.51 & 80.27 & 79.86 & 80.00 & 81.94 & 82.04 \\
\hline Average & 81.70 & 81.76 & 79.67 & 82.59 & 82.595 & 85.70 \\
\hline
\end{tabular}

UMCS@ is multiple of K-means + K-medians. UMCS\# is multiple of K-medians + Kohonen. UMCS* is multiple of K-means + K-medians + Kohonen. 
Table 7 Amount of improvements in the MA

\begin{tabular}{|c|c|c|c|}
\hline Class number & UMCS@-K-means (\%) & UMCS@-Kohonen (\%) & \\
\hline \multicolumn{4}{|c|}{ Improvement of UMCS@ over each of K-means and Kohonen } \\
\hline 1 & -0.98 & +2.53 & \\
\hline 2 & -1.53 & +6.59 & \\
\hline 3 & +2.39 & +7.90 & \\
\hline 4 & -3.08 & +3.04 & \\
\hline 5 & +0.97 & +0.48 & \\
\hline 6 & +1.33 & +0.59 & \\
\hline 7 & +6.57 & +1.11 & \\
\hline 8 & +0.49 & +0.14 & \\
\hline Average & +0.77 & +2.79 & \\
\hline \multicolumn{4}{|c|}{ Improvement of UMCS\# over each of K-medians and Kohonen } \\
\hline 1 & +0.15 & +4.01 & \\
\hline 2 & -2.70 & +3.23 & \\
\hline 3 & -0.44 & +1.82 & \\
\hline 4 & +0.68 & +6.44 & \\
\hline 5 & +1.94 & +0.48 & \\
\hline 6 & +0.50 & +3.90 & \\
\hline 7 & +4.83 & +1.39 & \\
\hline 8 & +1.67 & +2.08 & \\
\hline Average & +0.829 & +2.92 & \\
\hline \multicolumn{4}{|c|}{ Improvement of UMCS* over each of K-means, K-medians and Kohonen } \\
\hline Class number & UMCS*-K-means (\%) & UMCS*-K-medians (\%) & UMCS*-Kohonen (\%) \\
\hline 1 & +2.64 & +2.33 & +6.19 \\
\hline 2 & +1.26 & +2.39 & +8.32 \\
\hline 3 & +4.55 & +7.80 & +10.06 \\
\hline 4 & +3.34 & +4.70 & +10.46 \\
\hline 5 & +2.96 & +3.93 & +2.47 \\
\hline 6 & +8.51 & +4.37 & +8.77 \\
\hline 7 & +8.36 & +6.34 & +2.90 \\
\hline 8 & +2.53 & +1.77 & +2.18 \\
\hline Average & +4.27 & +3.70 & +6.41 \\
\hline
\end{tabular}

A: Improvement of UMCS@ over each of K-means and Kohonen. B: Improvement of UMCS\# over each of K-medians and Kohonen. C: Improvement of UMCS* over each of K-means, K-medians and Kohonen.

improvements in the class and average MA made by each of the multiple classifiers over their belonging individual classifiers. The best improvements were achieved by UMCS* over each of the individual classifiers. The amounts of these improvements are 4.27, 3.70 and $6.41 \%$ over each of K-means, K-medians and Kohonen classifiers respectively. These improvements are equivalent to areas of 3.37, 2.92 and $5.1 \mathrm{~km}^{2}$. Whereas the average improvements made by UMCS@ and UMCS\#, over each of the individual classifiers were much less. For UMCS@ the improvement over K-means and Kohonen were 0.77 and $2.79 \%$ (equivalent to areas of 0.61 and 2.2 $\mathrm{km}^{2}$ ) and for UMCS\# the improvement over K-medians and Kohonen were 0.829 and $2.92 \%$ (equivalent to areas of 0.65 and $2.3 \mathrm{~km}^{2}$ ). However, for individual classes the maximum improvement achieved by UMCS* reached $8.51,7.80$ and $10.46 \%$ over each of K-means, K-medians and Kohonen classifiers respectively. While the improvements made by UMCS@ over K-means and Kohonen were 6.57 and $6.59 \%$ respectively and by UMCS\# over $\mathrm{K}$-medians and Kohonen were 4.83 and $6.44 \%$ respectively. These improvements indicate that when more classifiers are used in the multiple classifier system better improvement can be achieved. This concluded result has also been approved for the supervised multiple classifier system, [14]. 


\section{Conclusions}

Unsupervised classifiers (K-means, K-medians and Kohonen) representing two different approaches, classical and artificial neural network, they were integrated in a MCS the application of the system to satellite images has shown that the three classifiers may produce different results despite of being considered as closely related. The CDM, which is generated from the CCDM, is shown to be effective measure for competition during the process of classifier output integration. The way of using the record of this map makes the chance of tie cases occurrence as less as possible. For the area used in this study no tie cases have occurred. The implementation of the system does not need training data; however, the test data derived after classification are necessary only for the evaluation of the system performance. The application of the system using three individual classifiers achieved better performance than its application with only two individual classifiers. This indicates that contributing more individual classifiers will make the made-off MCS more efficient.

However, this study may represent the beginning but important step in the direction of multiple classifier approach for unsupervised classification of remote sensing data and lots of work may bb required to put the approach steps further.

\section{Competing interests}

The author declares that he has no competing interests.

\section{Acknowledgement}

This study was supported by the University of Duhok / Faculty of Science as a part of the scientific research plan of the Physics Department for the year $2010 / 2011$.

Received: 13 October 2011 Accepted: 9 July 2012

Published: 31 July 2012

\section{References}

1. C Kamusoko, M Aniya, Hybrid classification of Landsat data and GIS for land use/cover change analysis of the Bindura district. Zimbabwe. Int. J. Remote. Sens. 30(1), 97-115 (2009)

2. U Kumar, SK Raja, C Mukhopadhyay, TV Ramachandra, Hybrid Bayesian Classifier for Improved Classification Accuracy. IEEE Trans. Geosci. Remote. Sens. Let. 8(3), 474-477 (2011)

3. G Palubinskas, M Datcu, R Pac, Clustering algorithms for large sets of heterogeneous remote sensing data. IEEE Geosci. Remote. Sens. 3, 1591-1593 (1999). Proceedings of International Symposium, IGARSS '99

4. PM Mather, B Tso, M Koch, An Evaluation of Landsat TM Spectral data and SAR-Derived Textural Information for Lithological Discrimination in the Red Sea Hills. Sudan, INT. J. Remote. Sens. 19(4), 587-607 (1998)

5. ML Goncalves, MLA Netto, JAF Costa, J Zullo Júnior, An unsupervised method of classifying remotely sensed images using Kohonen self organizing maps and agglomerative hierarchical clustering methods. Int. J. Remote. Sens. 29(11), 3171-3207 (2008)

6. F Anthony, D lliyana, AG Klein, JR Jensen, Self-Organizing Map-based Applications in Remote Sensing, Self-Organizing Maps, in InTech, ed. by G.K. Matsopoulos (2010). Available from: http://www.intechopen.com/articles/ show/title/self-organizing-map-based-applications-in-remote-sensing. ISBN 978-953-307-074-2

7. M Awad, An Unsupervised Artificial Neural Network Method for Satellite Image Segmentation. Int. Arab. J. Inf. Technol. 7(2), 199-205 (2010)
8. A AK Tahir, A multiple Classifier System for supervised classification of remotely sensed data. J. Univ. Duhok 14(1), 260-273 (2011)

9. U Maulik, D Chakraborty, A Robust Multiple Classifier System for Pixel Classification of Remote Sensing Images. J. Fundam. Inf. 101(4), 286-304 (2010)

10. MT El-Melegy, SM Ahmed, Neural networks in multiple classifier system for remote sensing image classification. Studies in Fuzziness and soft computing 210, 65-94 (2007)

11. PC Smits, Multiple classifier systems for supervised remote sensing image classification based on dynamic classifier selection. IEEE Trans. Geosci. Remote. Sens. 40(4), 801-813 (2002)

12. M Sabourin, A Mitiche, D Thomas, G Nagy, Classifier Combination of hand printed digit recognition, Proceeding of Second International Conference on Document Analysis and Recognition (IEEE conference publications, Tsukuba Saenie City, Japan, 1993), pp. 163-166. doi:10.1109//CDAR.1993.395796

13. PS Bradley, OL Mangasarian, WN Street, Clustering via Concave Minimization, in Advances in Neural Information Processing Systems, ed. by MC Mozer, Ml Jordan, T Petsche, vol. 9 (MIT Press, Cambridge, MA, 1997), pp. 368-374

14. JA Benediktsson, J Chanussot, M Fauvel, Multiple classifier systems in remote sensing: from basics to recent developments, Proceedings of the 7th international conference on Multiple classifier systems (Springer-Verlag, Berlin, Heidelberg, 2007), pp. 501-512

doi:10.1186/1687-6180-2012-165

Cite this article as: Tahir: Integrating artificial neural network and classical methods for unsupervised classification of optical remote sensing data. EURASIP Journal on Advances in Signal Processing 2012 2012:165.

\section{Submit your manuscript to a SpringerOpen ${ }^{\circ}$ journal and benefit from:}

- Convenient online submission

- Rigorous peer review

- Immediate publication on acceptance

- Open access: articles freely available online

- High visibility within the field

- Retaining the copyright to your article

Submit your next manuscript at $\gg$ springeropen.com 